\title{
SOCIETY NEWS
}

The Annual Meeting was held at Weyburn 10-12 October 1986. Among the special guests at this meeting was Dr. Geoff Holroyd, of the Canadian Wildlife Service in Edmonton. Geoff spoke and answered questions on endangered species and endangered species programs across Western Canada.

Perhaps the highlight of the meeting was Dr. David Henry's after dinner presentation on the Red Fox. David, author of the just published book, Red Fox: the catlike canine, used slides and film to give us an intimate look at the lives and behaviour of the Red Foxes of Prince Albert National Park.

Sheina Wait became the first two-time winner of the Larry Morgotch Award. Her winning entry in this competition included excellent photographs of Moose Jaw's Burrowing Owls.

Shirley Brunt was selected to receive the Cliff Shaw Award, for her article "Owls in our farmyard," in the June 1986 issue of Blue Jay. She described some very interesting behaviour of young Great Horned Owls.

The Conservation Award was presented to Connie Gramiak of Hafford, and members of the Redberry Environmental Group, in acknowledgement of their continuing efforts to preserve the unique value of Redberry Lake and its shores for wildlife. They have formed the major opposition to a cottage-resort development planned for the environmentally fragile southwest corner of the lake. Their efforts resulted in an Environmental Impact Study, and the subsequent denial of the developer's application (currently being appealed).
The islands and waters of Redberry were designated a Federal Bird Sanctuary in 1915. The Canadian Wildlife Service ranks this as the fourth most important of its 15 Saskatchewan sanctuaries. Some of the nesting islands are used by protected species. Saskatchewan has nearly half of the world's white pelican population and about $40 \%$ of the Piping Plover population. Both these species breed on this lake (pelicans on the islands, plovers on the shore). The Whitewinged Scoter population is believed to bethe largest breeding concentration of the species in North America. Research on Redberry's bird populations has resulted in one of the longest continuous bodies of data in the country, dating from the mid 1950s. The sanctuary status, however, cannot even protect the birds from harassment without some type of enforcement, and the populations are even now being affected by increased recreational use of this lake. 
SASKATCHEWAN NATURAL HISTORY SOCIETY

ASSETS:

Cash on hand (bookshops)

Bank - Current

Cirrent (looshshop)

Saving

baving (bookshop)

Investmente - Regular Term Life Membership - Mantey Catlin berpuest

stock on hand (bookinhop)

Arcounts Receivable

Customer acrounts receivable

less customer prepaid

Supplier prepaid accounts

TOTAL ASSETS

LIABILITIES:

Accounts pryable

Prepaid trom ST

Education tax payalole

Honorarium payable

TRUST ACCOUNTS

Habitat Conservation - opening bal. lese grant Loggerhead Shrike plus net donations s income plus bookshop earnings

Heritage Marsh - opening balance plus donations

less 1986 contribution

NCC Ecological

Reserve for computer purchase

Reserve tor Spec. Publ. opening

less expense(Bird Find.Regina) plus donation

CWS Trust Account re:Webb-opening plus 1986 income

Lite Membership Trust Fund

Mantey Callin Bequest Fund

\section{PROJECTS}

Burrowing (Owt Research - open. bal. less transter to end. p).

IBP Research - opening balance less expense's

plus income - sale of reports less cost reprinting report

Screech ()wl Reasearch - open bat plus return unexpended funds lexs transfer to end. ypp.

Endangered species Research -open plus wage \& WWF grants plus transterred funds plus ST Grant less expenses + wages plus donations plus income contributions

Yorkton Project-opening bal. - less expense's to date

Interpretive Programs - ST Grant less expenors

Critical Witdlife - ST Grant krus expensers

Floral Protection - ST Grant

Natural + listory Classen - expensens plus ST Grant

\section{NET WORTH:}

()pening balance

plus tour income (previously unrecorded) plus net gain
$\$ \quad 40.00$

$7,083.74$

$3,298.64$

$2,818.47$

1.08

$81,009.76$

$19,449.01$

$118,603.72$

7.665 .93

$3,850.00$

1.750 .63

289.63

$1,461.00$

233.79

$\$ 242,015.14$

$4,453.00$

10.150 .00

29.29

$2,972.27$

$6,356.91$

300.00

$1,815.87$

$3,500.00$

$3,254.88$

$2,440.00$

$5,000.00$

694.88

$1,050.00$

$1,706.50$

$4,775.00$

$2,628.43$

19.549 .01

118.603 .72

48.34

48.34

$4,860.00$

$4,760.00$

600.00

586.23

0.00

1.252 .86

$1,252.86$

$4,000.00$

$2,798.00$

$1,301.20$

$8,300.00$

$14,519.96$

22.05

$2,000.00$

$25,630.00$

$22,901.41$

3.400 .00

178.74

$12,000.00$

$8,000.00$

938.85

$1,200.00$

0.00

113.77

0.00

$3,901.29$

$2,728.59$

3.221 .26

$4,000.00$

$1,100.00$

261.15

$3.7483 .2^{\circ}$

$4,063.80$

892.05 
SASKATCHEWAN NATURAL HISTORY SOCIETY

INCOME:

Operating Statement for the year ended September 30, 1986

Membership

Sustaining and Patron

Donations - general

Interest - received

Tour income

Sales Special Publication

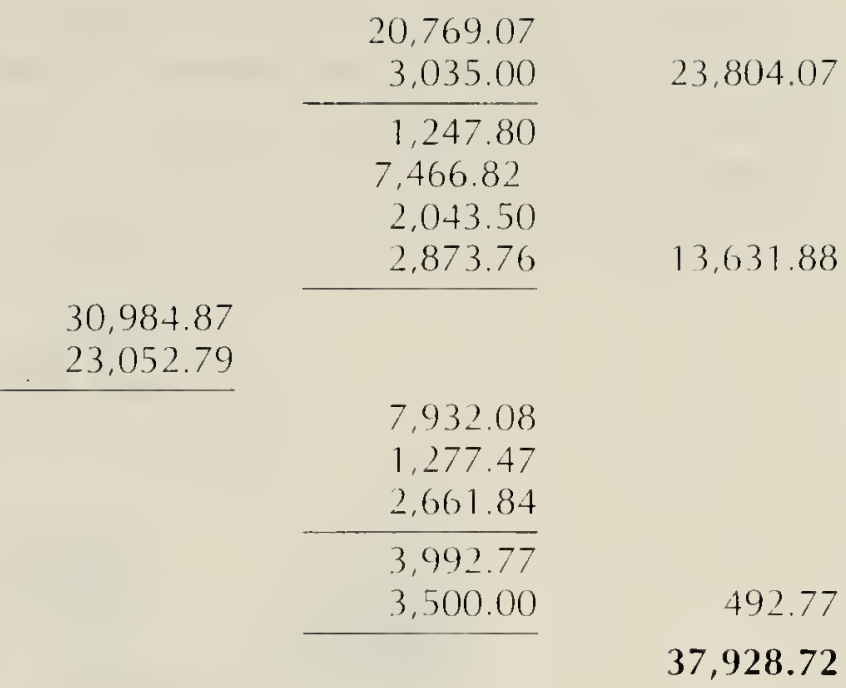

Bookshop sales

less cost of sales

$30,984.87$

gross profit

$23,052.79$

less operating expenses

less honorarium $(40 \%)$

Net bookshop profit

less contribution to habitat conservation

\section{TOTAL INCOME}

\section{EXPENSES:}

Advertising and Promotion less ST grant

Conservation Activities-South Moresby - Ellis Bird Farm

-computerisation of Saskatoon bird records

-Fort Qu'Appelle Herbarium purchase

-Northern Forest OwI Symp.

-Hidden Valley

-Endangered spp. research

Audit Costs

Bank charges

Computer expense

Employment expense

Honoraria

Meeting - annual (profit)

Meeting Expenses - board

Memberships (Canadian Nature Fed.)

Misc. Office \& Admin.

Office equipment

Office Supplies \& Stationary less ST grant

Postage \& Express

Blue lay - printing

$$
\text { - mailing }
$$

Blue lay New - printing

$$
\text { - postage }
$$

- lesas ST grant

598.59

1000.00

200.00

200.00

$5,000.00$

$5,000.00$

250.00

$4,000.00$

$2,000.00$

$16,650.00$

37.50

27.95

739.00

253.14

900.00

$(287.48)$

295.17

100.00

831.18

225.91

$1,045.91$

500.00

545.91

$2,045.45$

$14,456.09$

495.48

2.178 .30

3.117 .28

$3,000.00$

117.28

Cost of Society publ.

(Taxon. Remindier)

Qu'Appelle Valley Birds
lese donation

88.50

-Eskimo Curlew

lese Env.Can.contr.

less donation

443.9()

$250.00 \quad 193.90$

$9,705.90$

7.400 .00

2.500 .00

88.30

(194.10)

1.670.12

1.521 .98

$4.000 .0(0)$

$(2,478,(1) 2)$

$11,540.80$

13.000 .000

$(1.45() .2())$ 


\section{ATTENTION SASKATCHEWAN NATURALISTS!}

Be sure to attend the SNHS Annual Summer Meet in $\mathbf{1 9 8 7}$ being held in conjunc tion with the Canadian Nature Federation Conference, hosted by the Saskatoon Natural History Society, at the University of Saskatchewan, 5-8 June. We will be celebrating the 100th Anniversary of the Last Mountain Lake Sanctuary -- the oldest in Canada, and 100 years of conservation in Saskatchewan.

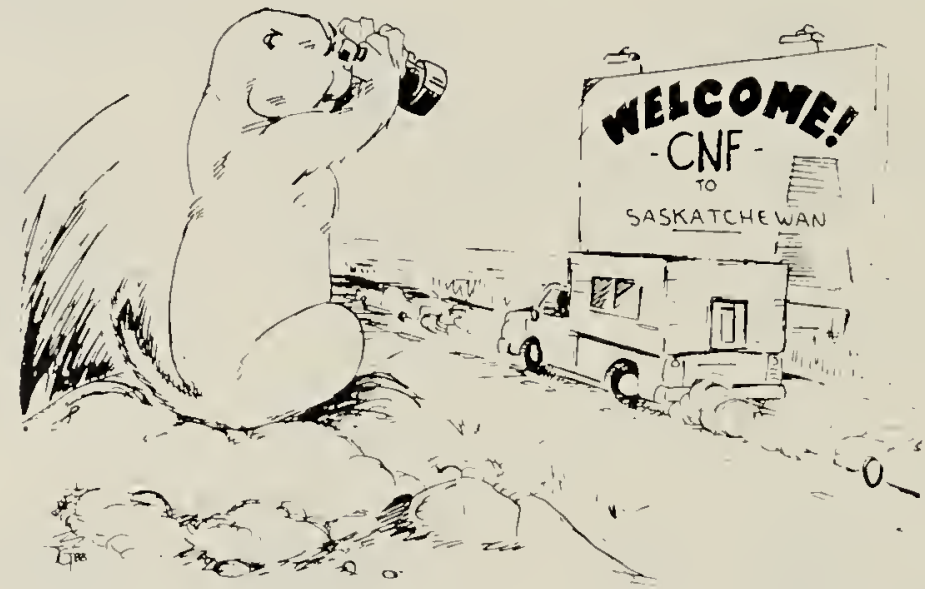

16th Annual CNF Conference, U. of S., Saskatoon, 5-8 june 1987

\section{CALENDAR OF EVENTS}

June 2-5: Pre-conference field trips

June 4 : Slides, films (evening)

June 5 : Orientation, annual meeting (afternoon)

: Wine and cheese (evening)

June 6 : Symposium (all day)

Banquet (evening)

June 7 : Field trips

June 8 : Last Mountain Lake 100 year celebration

June 8-15: Post-conference field trips

\section{FIELD TRIPS*}

A. Turtle Lake 2-5 June

B. Blackstrap Park 4 June

C. Batoche Historic Park 7 June

D. Beaver Creek 7 June

E. Beechy Grasslands 7 June

F. Last Mountain Lake 8 June

G. Duck Mountain 8-10 June ** 150

H. Duck Mt.-Churchill 8-15 June 590

I. Cypress Hills 8-12 June

J. Qu'Appelle Valley 8-10 June 150

K. Pike Lake 9 June

L. Meewasin Valley Nature Walk free

* costs estimated

** Trips G-J go by way of Last Mt. Lake

\section{SYMPOSIUM}

Theme of symposium -- "A Century of Wildlife Conservation in Saskatchewan." Topics of the speakers include Last Mountain Lake, birds in Saskatchewan, land use, naturalists, Cypress Hills and Grasslands National Park.

AND THERE'S MORE . . .

Early morning nature walks

Blue Jay and Nature Canada bookshops

Nature art show and nature exhibits Banquet and wine and cheese party

Accommodation available at University Residences

REGISTRATION fee is $\$ 30$ or less

Sign up early for field trips -- space is limited.

FOR DETAILED INFORMATION PACKET AND REGISTRATION FORMS write now to: CNF Conference 1987, Box 5094, Saskatoon, Saskatchewan. S7K 4E4

or telephone (306) 652-5970 $8 \mathrm{am}-8 \mathrm{pm}$ CST and leave your name and address. 